\title{
Changes in the incidence of seasonal influenza in response to COVID-19 social distancing measures: an observational study based on Canada's national influenza surveillance system
}

\section{Andrew Pierce ( $\sim$ piercajs@myumanitoba.ca )}

National Collaborating Centre for Infectious Diseases, Faculty of Health Sciences, Max Rady College of Medicine, University of Manitoba, Winnipeg, Manitoba, Canada https://orcid.org/0000-0001-8577-0589 Margaret Haworth-Brockman

National Collaborating Centre for Infectious Diseases, Faculty of Health Sciences, Max Rady College of Medicine, University of Manitoba, Winnipeg, Manitoba, Canada https://orcid.org/0000-0002-9196-2242 Diana Marin

Facultad de Medicina, Universidad Pontificia Bolivariana https://orcid.org/0000-0002-4715-8388

\section{Zulma V Rueda}

Facultad de Medicina, Universidad Pontificia Bolivariana https://orcid.org/0000-0001-6342-1812

\section{Yoav Keynan}

National Collaborating Centre for Infectious Diseases, Faculty of Health Sciences, Max Rady College of Medicine, University of Manitoba, Winnipeg, Manitoba, Canada https://orcid.org/0000-0003-4948-4707

\section{Research Article}

Keywords: seasonal influenza, COVID-19, coronavirus, social distance, Canada

Posted Date: October 22nd, 2020

DOI: https://doi.org/10.21203/rs.3.rs-93953/v1

License: (c) (i) This work is licensed under a Creative Commons Attribution 4.0 International License. Read Full License

Version of Record: A version of this preprint was published at Canadian Journal of Public Health on May 28th, 2021. See the published version at https://doi.org/10.17269/s41997-021-00509-4. 


\section{Abstract}

Objectives: Seasonal influenza is an acute respiratory infection that presents a significant annual burden to Canadians and the Canadian health care system. Social distancing measures that were implemented to control the novel coronavirus outbreak were also investigated for their ability to lessen the incidence of seasonal influenza.

Methods: We conducted an ecological study using data from Canada's national influenza surveillance system to investigate whether social distancing measures to control COVID-19 reduced the incidence of seasonal influenza. Data taken from three separate time frames facilitated analysis of the 2019-20 influenza season prior to, during, and following the implementation of COVID-19 related measures and enabled comparisons to the same time periods during three preceding flu seasons. The incidence of specific influenza strains was of primary focus. Further analysis was performed to determine the number of new laboratory-confirmed influenza or influenza like illness outbreaks.

Results: Our results indicate a premature end to the 2019-20 influenza season, with a significantly fewer number of cases and outbreaks being recorded following the enactment of many COVID-19 social distancing polices. The incidence of influenza strains A (H3N2), A (unsubtyped), and B were all significantly lower at the tail-end of the 2019-20 influenza season, compared with preceding seasons.

Conclusion: Specific social distancing measures and behaviours may serve as effective tools to limit the spread of influenza transmission moving forward, as they become more familiar.

\section{Introduction}

Seasonal influenza (flu) is an acute respiratory infection caused by the Orthomyxoviridae family of influenza viruses (Infection Prevention and Control Canada 2020). It is often characterized by the sudden onset of cough, headache, fever, chills, myalgia, fatigue and a general feeling of malaise (Infection Prevention and Control Canada 2020). While forms of the illness can range from mild to severe, the influenza virus presents as a significant burden worldwide, accounting for up to 650, 000 respiratory deaths annually (luliano et al., 2018). Influenza, along with pneumonia, consistently remains one of the top ten leading causes of death in Canada and is responsible for nearly 12, 200 hospitalizations and 3 , 500 deaths each year (Public Health Agency of Canada Flu 2020). These figures are likely underestimated, as laboratory confirmation varies according to age and clinical context, and it remains challenging to determine the extent to which influenza directly or indirectly contributes to health conditions that influence overall mortality(National Institute on Ageing 2018).

Currently, the World Health Organization (WHO) recommends annual vaccination as the most effective way to prevent infection and severe outcomes caused by influenza viruses (World Health Organization 2020). This is especially true for those who may be particularly vulnerable to contracting severe forms of influenza, such as children under 5 , people over 65 , pregnant women, individuals living with chronic medical conditions, those who are otherwise immunocompromised, residents of long-term care facilities, 
and healthcare workers (Public Health Agency of Canada 2016). Vaccination, however, remains moderately effective as it is highly dependent on the match between the circulating strain of virus and the vaccine, as well as the uptake and characteristics of those being vaccinated. For example, in Canada, annual vaccine coverage among adults varies: vaccination coverage in the $2018-19$ season was $42 \%$; $38 \%$ in 2017-18, and 36\% in 2016-17, with higher rates in women (women make up $80 \%$ of the healthcare work force in Canada); (Public Health Agency of Canada 2019; Canadian Institute for Health Information 2017). Other public health recommendations to prevent the spread of influenza focus on improving personal hygiene, avoiding crowded places through the practice of social distancing, and the use of protective face masks. While adherence to these measures has proven to be limited in years past, they have garnered much greater attention this season (2019-20) as they were imposed to control the novel coronavirus outbreak.

COVID-19 is an acute respiratory syndrome caused by the newest member of the family of coronaviruses that has given rise to a recent global pandemic (World Health Organization 2020). The most common symptoms of COVID-19 often resemble those of the common cold and can range in severity from mild to severe(World Health Organization 2020). Both COVID-19 and the influenza virus are primarily respiratory illnesses that are highly transmittable via contact, droplets, and fomites. Thus, public health measures designed to control the spread of COVID-19 may have helped to lessen the spread and burden of the seasonal influenza virus. The goal of this study was to compare trends in the 2019-20 season of influenza in Canada to preceding years, and to determine if any changes in seasonal influenza coincide with specific policies and measures implemented by the Canadian government to limit the spread of COVID-19.

\section{Methods}

An ecological study was conducted using information regarding influenza incidence and associated morbidity and mortality in Canada. Data was obtained from FluNet, the WHO influenza surveillance database, and FluWatch, Canada's national surveillance system for weekly monitoring of the spread of influenza and other associated illnesses (Benchimol et al., 2015; Government of Canada 2020). FluWatch relies on a system of laboratories, hospitals, doctors' offices, and provincial or territorial ministries of health to collect data. Key parameters help inform policies to limit the spread of influenza. The seven components of influenza surveillance in Canada include: the geographic spread of influenza and influenza-like illness (ILI) activity; laboratory-confirmed detections; syndromic surveillance; outbreak surveillance; severe outcomes surveillance; strain characterization and antiviral resistance testing; and vaccine monitoring (Government of Canada 2019).

Of particular interest to this study was information characterizing the circulating strains of four influenza subtypes: A (unsubtyped), A (H3N2), A (H1N1), and B, and data classifying the new number of laboratoryconfirmed influenza or ILI outbreaks. These data were retrieved from weekly influenza reports across the last four flu seasons (2016-2020) and subsequently separated into three distinct time periods related to the onset of COVID-19 in Canada, and the ensuing policies implemented to limit its spread. Creating the 
three distinct time frames for each season made it possible to compare the 2019-20 flu season to the same time periods in preceding years. The first period of the flu season was assigned to epidemiological weeks 41 to 6 , spanning from the onset of flu reporting in October to the middle of February, prior to the initiation of measures intended to control COVID-19. Weeks 7 to 12 (mid-February to the end of March) of the 2019-20 flu season correspond to the period at which the majority of the COVID-19 related closures and policies were implemented across Canada. Lastly, weeks 13 to 18 (end of March to the beginning of May) were chosen to investigate if COVID-19 related measures had any influence on the incidence of the seasonal influenza during the time frame most often corresponding to the tail-end of the flu season.

Data were tabulated in Excel ${ }^{\circledR}$ and analyzed in STATA $\circledast$ version 15.0. Box-plots were used to provide a visual comparison of the incidence of influenza cases and outbreaks across designated seasons and time frames. Non-parametric tests including Kruskal-Wallis and Wilcoxon rank-sum were completed to compare the incidence and type of influenza cases across season years as well as the location and number of influenza-associated outbreaks. A bilateral $p$-value of $\leq 0.05$ was considered significant.

\section{Results}

Table 1 and Figures 1 and 2 show the incidence of positive influenza tests in four flu seasons according to influenza subtypes: A (unsubtyped), A (H3N2), A (H1N1), and B. No differences were found between the median values for $A$ (unsubtyped) over the past four seasons when comparing weeks 41 to 6 ( $p=$ 0.1607). From weeks 7 to 12 a significantly higher number of influenza $A$ (unsubtyped) cases were reported in 2020 compared to the preceding seasons $(p=0.0494)$. However, a significantly lower number

of $A$ (unsubtyped) cases were recorded in the final time period from weeks 13 to 18 in 2020 compared to other seasons $(p=0.0007)$. When comparing the results for influenza $A(H 3 N 2)$, a significantly lower number of influenza cases were documented during the time frames spanning weeks 7 to 12 , and 13 to 18 in the 2020 flu season compared to other seasons $(p=0.0010, p=0.0003$, respectively) (Table 1 and Figure 1). Similar findings were reported for influenza $B$, with the 2020 season revealing a significantly lower number of positive tests between weeks 13 to $18(p=0.0019)$ (Figure 2). Significant differences were also noted in the median recorded values for the A (H1N1) strain, however the 2020 season was found to have a significantly higher incidence of cases between weeks 7 to 12 over the preceding seasons $(p=0.0003)$. For each strain during the 2020 flu season a significantly lower number of positive influenza tests were recorded between weeks 13 to 18 than the two preceding time frames.

Analysis was also undertaken to assess the number and location of new laboratory-confirmed influenza cases by week (Table 2 and Figure 3). No significant differences were recorded in the number of outbreaks occurring in hospitals or long-term care facilities between weeks 41 to 6 of 2019-20 compared with the three preceding flu seasons. However, during weeks 7-12 a significantly lower number of hospital associated outbreaks were reported in 2020 compared to the preceding seasons $(p=0.0042)$. Similar results were also observed for weeks 13-18 in both long-term care facilities and facilities classified as other ( $p=0.0035, p=0.0014$, respectively). Lastly, the number of outbreaks in 2020 at each type of facility was significantly lower during weeks 13 to 18 than the two preceding time frames. 


\section{Discussion}

Seasonal influenza is a serious threat globally, from both a health and economic perspective(Public Health Ontario 2017). Complications resulting from influenza can further lead to increased hospitalizations, higher mortality rates, and a decrease in economic productivity(National Institute on Ageing 2018). In Canada, women and men over 65 are at greatest risk for suffering from severe cases of influenza due to the higher prevalence of chronic underlying health conditions. Notwithstanding the immense burden that the flu presents annually, it is not perceived as a significant threat to the population as the majority of cases result in mild to moderate forms of the illness. While the Canadian government endorses vaccination as the best way to prevent the flu and recommends that $80 \%$ of high-risk adults get vaccinated, data from the 2018-19 season indicates that only $70 \%$ of adults over age 65 and $43 \%$ of adults aged 18-64 with a chronic medical condition received vaccination (Canadian Institute for Health Information 2017). In addition, roughly four out of ten respondents in a survey believed that vaccination was not effective against the flu (41\%) and a similar number believed vaccination could lead to acquiring the flu (43\%) (Canadian Institute for Health Information 2017). Despite educational campaigns and attempts to increase access to vaccination at most pharmacies and public health clinics, vaccine coverage has not reached targets set by the Canadian government. This season, however, with the outbreak of COVID-19, there is a unique opportunity to investigate how social distancing measures could affect the incidence of seasonal influenza.

In the present study, a significantly lower number of influenza cases were reported in Canada in 2020 between weeks 7 to 18 for influenza A (H3N2) and B viruses. A high number of cases caused by influenza A (unsubtyped) was reported in weeks 7-12, with a precipitous decline during weeks 13 to 18 . The weeks during which fewer cases were reported roughly correspond to the implementation of social distancing measures to control the spread of COVID-19 in Canada. As depicted in Figure 4, most of the initial warnings related to travel occurred near the middle of February around week 7 . This was then followed by the enactment of policies designed to prevent human to human transmission of the virus towards the middle of March. Thus, the lower number of cases reported for influenza strains A (H3N2) and B could reflect the initial warnings related to COVID, while the lower incidence of cases following week 13 may be more reflective of the actual policies implemented. Kuo et al. observed similar trends in Taiwan (Kuo, Shih, Chien, \& Hsiung, 2020). This theory is substantiated by the fact that the number of influenza cases reported in 2020 for each strain was significantly lower during weeks 13 to 18 than either of the previously recorded time frames spanning from weeks 41 to 12 . As a result, it was determined that the 2020 flu season ended abruptly in week 12, approximately two months earlier than in each of the past three seasons. This change in course could be the result of interrupted influenza reporting as several countries around the globe have had to change their focus or repurpose their influenza surveillance systems to also track the COVID-19 virus (World Health Organization 2020; Government of Canada 2020). However, reports by the WHO suggest that the number of influenza tests administered in Canada actually increased prior to and during the onset of COVID-19(World Health Organization 2020). In fact, the time period at which the incidence of influenza cases decreased most significantly was also the same time during which the most tests were processed. 
The number of laboratory-documented outbreaks also appeared to be related to the onset of COVID-19 measures. Significantly lower numbers of outbreaks in several types of facilities were documented in 2020 compared to previous seasons. In addition, the number of outbreaks reported in each facility was found to be significantly less between weeks 13 to 18 than the previously reported time periods. These findings correspond to the approximate dates at which key closures or restrictions were implemented by various levels of government (Figure 4). For example, the closure of public schools in the province of Ontario and the restrictions imposed on visiting seniors in long-term care facilities in two provinces, Ontario and Quebec, align with decreased documented outbreaks in those particular settings. While the exact influence of social distancing measures on influenza outbreaks is difficult to quantify, it would be useful to have a better understanding about how human behaviours could be modified to more effectively protect those who are vulnerable.

This study is not without limitations. First, Canada's geographic landscape makes it difficult to conduct influenza surveillance as the timing and burden of influenza activity differs considerably across the country(Public Health Agency of Canada 2019). Not all health ministries and hospitals participate in flu surveillance which can present challenges to having a comprehensive and representative dataset for analysis. Furthermore, FluWatch data are not disaggregated by sex and age, which limits our analyses to generalizations across the population, although there may have been quite different results within population groups. High variability between annual influenza seasons makes comparison difficult. We endeavoured to overcome this limitation by performing the comparison across several influenza seasons, and incorporate weeks 41 to 6 into our analysis, before the first COVID-19 case diagnosed in Canada (Marchand-Senécal et al., 2020). Additionally, many people with mild to moderate illnesses or ILI may have avoided clinics, thus limiting the number of influenza tests ordered and documented. This would have been especially true during the first peak of the COVID-19 pandemic when Canadians were strongly encouraged to self-isolate and remain socially distant.

\section{Conclusion}

The measures and policies that were implemented in Canada to limit the spread of COVID-19 appear to have led to a corresponding decrease in the incidence of seasonal influenza virus. However, specific policies that were effective controlling the pandemic, for example school closures and travel restrictions, are unlikely to be implemented annually. In contrast, more simple behaviours related to social distancing, limited gathering sizes, and improving personal hygiene are still effective in controlling the spread of influenza and may also be more readily adopted (Chiu et al., 2020; Fong et al., 2020). Ultimately, further research will be necessary to determine how we can effectively use this information to inform policies and behaviours that lessen the burden of seasonal influenza for Canadians and upon our health care system.

\section{References}


Benchimol, E. I., Smeeth, L., Guttmann, A., Harron, K., Moher, D., Petersen, I., ... Langan, S. M. (2015). The REporting of studies Conducted using Observational Routinely-collected health Data (RECORD) Statement. PLOS Medicine, 12(10), e1001885. https://doi.org/10.1371/journal.pmed.1001885

Canadian Institute for Health Information. (2017). Gender, workforce and health system change in Canada. Retrieved from

https://www.who.int/hrh/Oral-Gender-equity-and-womens-economic-empowerment-Porter-andBourgeault-16Nov-17h30-18h30.pdf?ua=1.Accessed August 14, 2020,

Chiu, N.-C., Chi, H., Tai, Y.-L., Peng, C.-C., Tseng, C.-Y., Chen, C.-C., ... Lin, C.-Y. (2020). Wearing masks, hand hygiene, and social distancing contribute to the decrease of influenza and all-cause pneumonia in coronavirus pandemic: a retrospective national epidemiological surveillance study. Journal of Medical Internet Research. https://doi.org/10.2196/21257

Fong, M. W., Gao, H., Wong, J. Y., Xiao, J., Shiu, E. Y. C., Ryu, S., \& Cowling, B. J. (2020, May 1). Nonpharmaceutical measures for pandemic influenza in nonhealthcare settings-social distancing measures. Emerging Infectious Diseases, Vol. 26, pp. 976-984. https://doi.org/10.3201/eid2605.190995

Government of Canada. (2020). FluWatchers. Retrieved from

https://www.canada.ca/en/public-health/services/diseases/flu-influenza/fluwatcher.html.Accessed August 15, 2020

Government of Canada. (2019). Overview of influenza monitoring in Canada. Retrieved from https://www.canada.ca/en/public-health/services/diseases/flu-influenza/influenza-surveillance/aboutfluwatch.html.Accessed August 11, 2020

Infection Prevention and Control Canada. (2020). Seasonal Influenza, Avian Influenza and Pandemic Influenza. Retrieved from

https://ipac-canada.org/influenza-resources.php.Accessed July 14, 2020

Iuliano, A. D., Roguski, K. M., Chang, H. H., Muscatello, D. J., Palekar, R., Tempia, S., ... Mustaquim, D. (2018). Estimates of global seasonal influenza-associated respiratory mortality: a modelling study. The Lancet, 391(10127), 1285-1300. https://doi.org/10.1016/S0140-6736(17)33293-2

Kuo, S. C., Shih, S. M., Chien, L. H., \& Hsiung, C. A. (2020). Collateral Benefit of COVID-19 Control Measures on Influenza Activity, Taiwan. Emerging Infectious Diseases, 26(8), 1928-1930. https://doi.org/10.3201/eid2608.201192

Marchand-Senécal, X., Kozak, R., Mubareka, S., Salt, N., Gubbay, J. B., Eshaghi, A., ... Leis, J. A. (2020). Diagnosis and Management of First Case of COVID-19 in Canada: Lessons applied from SARS. Clinical 
Infectious Diseases: An Official Publication of the Infectious Diseases Society of America. https://doi.org/10.1093/cid/ciaa227

National Institute on Ageing. (2018). The Underappreciated Burden of Influenza Amongst Canada's Older Population. And What We Need to Do About It. Retrieved from

https://www.ryerson.ca/content/dam/nia/white-papers/burden-of-influenza.pdf.Accessed July 14, 2020.

Public Health Agency of Canada. (2016). Canadian Immunization Guide Chapter on Influenza and Statement on Seasonal Influenza Vaccine for 2016-2017. Retrieved from https://www.canada.ca/en/public-health/services/immunization/national-advisory-committee-onimmunization-naci/canadian-immunization-guide-chapter-on-influenza-statement-on-seasonal-influenzavaccine-2016-2017-advisory-committee-statement.html\#t1.Accessed August 13, 2020,

Public Health Agency of Canada. (2019). Flu (influenza): For health professionals. Retrieved, from https://www.canada.ca/en/public-health/services/diseases/flu-influenza/healthprofessionals.html.Accessed July 14, 2020

Public Health Agency of Canada.(2019). Surveillance annex: Canadian Pandemic Influenza Preparedness: Planning Guidance for the Health Sector. Retrieved from https://www.canada.ca/en/public-health/services/flu-influenza/canadian-pandemic-influenzapreparedness-planning-guidance-health-sector/surveillance-annex.html\#a22.Accessed July 15, 2020

Public Health Agency of Canada. (2019). Vaccine uptake in Canadian Adults 2019. Retrieved from https://www.canada.ca/en/public-health/services/publications/healthy-living/2018-2019-influenza-fluvaccine-coverage-survey-results.html.Accessed August 10, 2020

Public Health Ontario. (2017). The relationship between influenza medical risk factors and age. (2017). Retrieved from

https://www.publichealthontario.ca/-/media/documents/I/2017/influenza-risk-factors-age-technical.pdf? la=en.Accessed July 24, 2020

World Health Organization. (2020). Vaccine Use. Retrieved from

https://www.who.int/influenza/vaccines/use/en/.Accessed July 14, 2020

World Health Organization. (2020). 2019-2020 influenza season: repurposing surveillance systems for COVID-19. Retrieved from

https://www.euro.who.int/en/health-topics/communicablediseases/influenza/news/news/2020/5/20192020-influenza-season-repurposing-surveillance-systemsfor-covid-19.Accessed Aug 10, 2020 
World Health Organization. (2020). Q\&A on coronaviruses (COVID-19). Retrieved from https://www.who.int/emergencies/diseases/novel-coronavirus-2019/question-and-answers-hub/q-adetail/q-a-coronaviruses.Accessed July 22, 2020

World Health Organization. (2020). WHO FLUMART OUTPUTS. Retrieved from https://apps.who.int/flumart/Default?ReportNo=12.Accessed July 23, 2020

\section{Tables}

Table 1. Number of positive influenza tests in Canada categorized by subtype and weekly timeframe for influenza seasons, 2016-17 to 2019-20

\begin{tabular}{|c|c|c|c|c|}
\hline $\begin{array}{r}\text { Influenza type } \\
\text { Weeks }\end{array}$ & $\begin{array}{c}2016-2017 \\
\text { Median }\left(\mathrm{P}_{25}-\mathrm{P}_{75}\right)\end{array}$ & $\begin{array}{c}2017-2018 \\
\text { Median }\left(\mathrm{P}_{25}-\mathrm{P}_{75}\right)\end{array}$ & $\begin{array}{c}2018-2019 \\
\text { Median }\left(\mathrm{P}_{25}-\mathrm{P}_{75}\right)\end{array}$ & $\begin{array}{c}2019-2020 \\
\text { Median }\left(\mathrm{P}_{25}-\mathrm{P}_{75}\right)\end{array}$ \\
\hline \multicolumn{5}{|l|}{ A (unsubtyped) } \\
\hline $41-6$ & $226.5(31-1222)$ & $523(71-1675)$ & $1042.5(244-1613)$ & $372(57-1620)$ \\
\hline $7-12$ & $889.5(629-1291)$ & $1023(807-1437)$ & $1075.5(990-1116)$ & $1754.5(1552-1772)$ \\
\hline $13-18$ & $191.5(113-273)$ & $336(215-436)$ & $685(448-939)$ & $9.5(4-48)$ \\
\hline \multicolumn{5}{|l|}{ A H3N2 } \\
\hline $41-6$ & $440.5(140-1225)$ & $506(153-808)$ & $35(18-93)$ & $77(43-139)$ \\
\hline $7-12$ & $812.5(347-935)$ & $265(246-279)$ & $310(236-357)$ & $72(58-76)$ \\
\hline $13-18$ & $66(47-134)$ & $105.5(62-143)$ & $284.5(244-387)$ & $1(0-5)$ \\
\hline \multicolumn{5}{|l|}{ A H1N1 } \\
\hline $41-6$ & $4(4-4)$ & $33(7-54)$ & $519.5(295-828)$ & $57.5(20-370)$ \\
\hline $7-12$ & $12(7-14)$ & $73(51-76)$ & $214.5(197-244)$ & $312(255-354)$ \\
\hline $13-18$ & $4(0-6)$ & $37(29-49)$ & $48.5(41-121)$ & $5(0-11)$ \\
\hline \multicolumn{5}{|l|}{ B } \\
\hline $41-6$ & $14.5(7-39)$ & $473(47-1802)$ & $15.5(8-40)$ & $495(39-1463)$ \\
\hline $7-12$ & $181(128-242)$ & $1714(1387-2057)$ & $67.5(40-90)$ & $1219.5(1015-1599)$ \\
\hline $13-18$ & $318.5(309-347)$ & $411(238-661)$ & $168(157-175)$ & $16.5(11-97)$ \\
\hline
\end{tabular}

P: percentile

Table 2. Number and location of new influenza outbreaks in Canada by weekly timeframe for four influenza seasons, $2016-17$ to $2019-20$ 


\begin{tabular}{lllll}
\hline $\begin{array}{l}\text { Setting } \\
\text { Weeks }\end{array}$ & $\begin{array}{l}\mathbf{2 0 1 6 - 2 0 1 7} \\
\text { Median }\left(\mathbf{P}_{\mathbf{2 5}}-\mathbf{P}_{\mathbf{7 5}}\right)\end{array}$ & $\begin{array}{l}\mathbf{2 0 1 7 - 2 0 1 8} \\
\text { Median }\left(\mathbf{P}_{\mathbf{2 5}}-\mathbf{P}_{\mathbf{7 5}}\right)\end{array}$ & $\begin{array}{l}\mathbf{2 0 1 8 - 2 0 1 9} \\
\text { Median }\left(\mathbf{P}_{\mathbf{2 5}}-\mathbf{P}_{\mathbf{7 5}}\right)\end{array}$ & $\begin{array}{l}\mathbf{2 0 1 9 - 2 0 2 0} \\
\text { Median }\left(\mathbf{P}_{\mathbf{2 5}}-\mathbf{P}_{\mathbf{7 5}}\right)\end{array}$ \\
\hline Hospitals & & & & \\
\hline $\mathbf{4 1 - 6}$ & $3(1-8)$ & $9(0-11)$ & $2.5(0-6)$ & $3(0-5)$ \\
\hline $\mathbf{7 - 1 2}$ & $4.5(3-7)$ & $8(8-8)$ & $8(6-10)$ & $1(1-2)$ \\
\hline $\mathbf{1 3 - 1 8}$ & $1(0-1)$ & $3(1-5)$ & $4(1-9)$ & $0(0-0)$ \\
\hline Long term & & & & \\
\hline $\mathbf{4 1 - 6}$ & $14.5(3-44)$ & $31(2-72)$ & $7.5(2-25)$ & $10(2-48)$ \\
\hline $\mathbf{7 - 1 2}$ & $32(28-38)$ & $46(44-60)$ & $32.5(27-39)$ & $31.5(20-34)$ \\
\hline $\mathbf{1 3 - 1 8}$ & $11.5(8-14)$ & $14(6-30)$ & $24.5(18-32)$ & $1.5(0-6)$ \\
\hline Other & & & & \\
\hline $\mathbf{4 1 - 6}$ & $3.5(0-13)$ & $16(4-28)$ & $3.5(1-7)$ & $4(1-15)$ \\
\hline $\mathbf{7 - 1 2}$ & $9(8-12)$ & $12(10-16)$ & $13(9-15)$ & $8(6-13)$ \\
\hline $\mathbf{1 3 - 1 8}$ & $6(6-7)$ & $6(4-8)$ & $11.5(8-13)$ & $1(0-2)$ \\
\hline
\end{tabular}

Figures

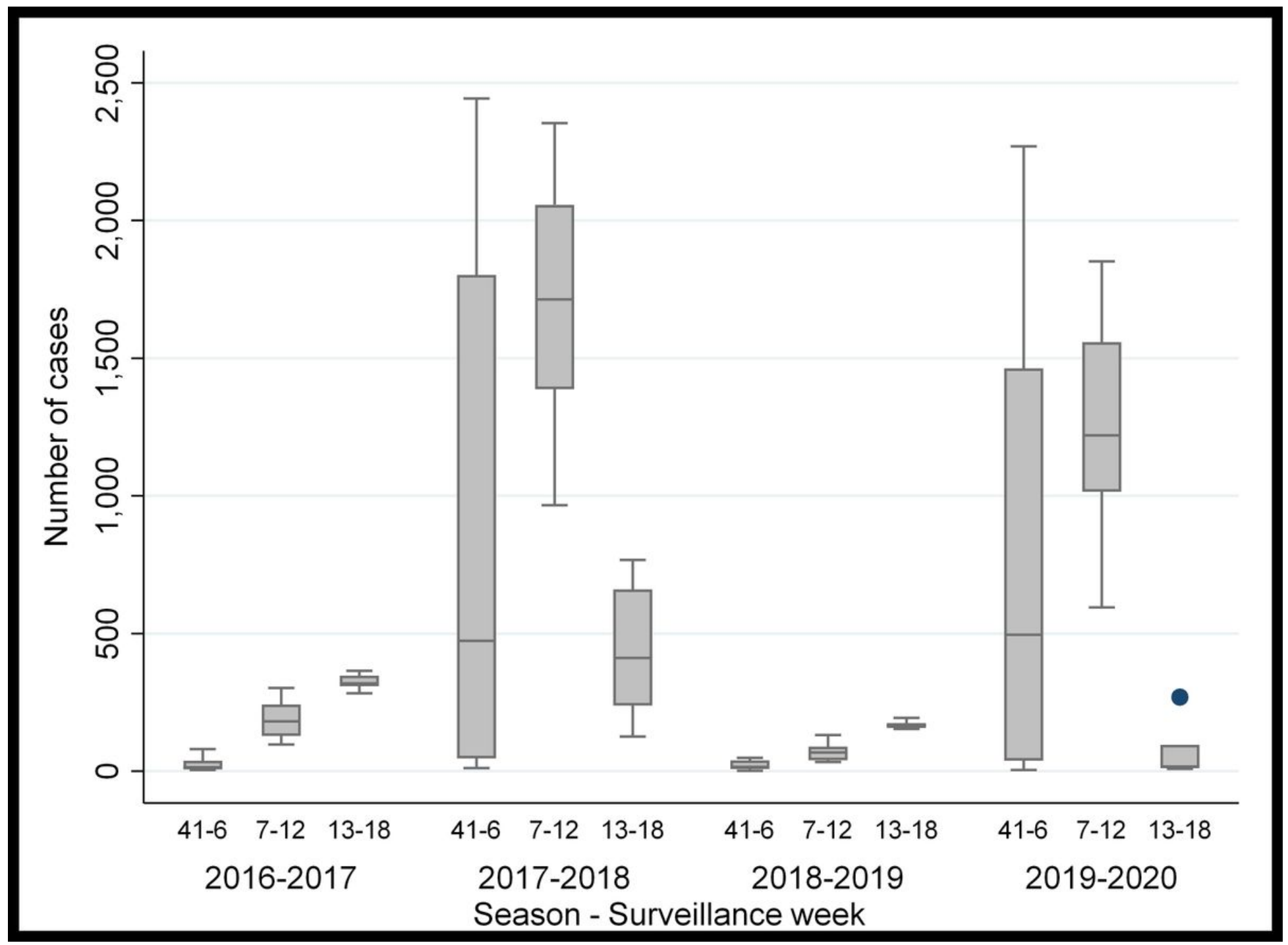


Figure 1

Box-plot of the number of positive influenza tests in Canada categorized by influenza A subtype and weekly timeframe for four influenza seasons, 2016-17 to 2019-20.

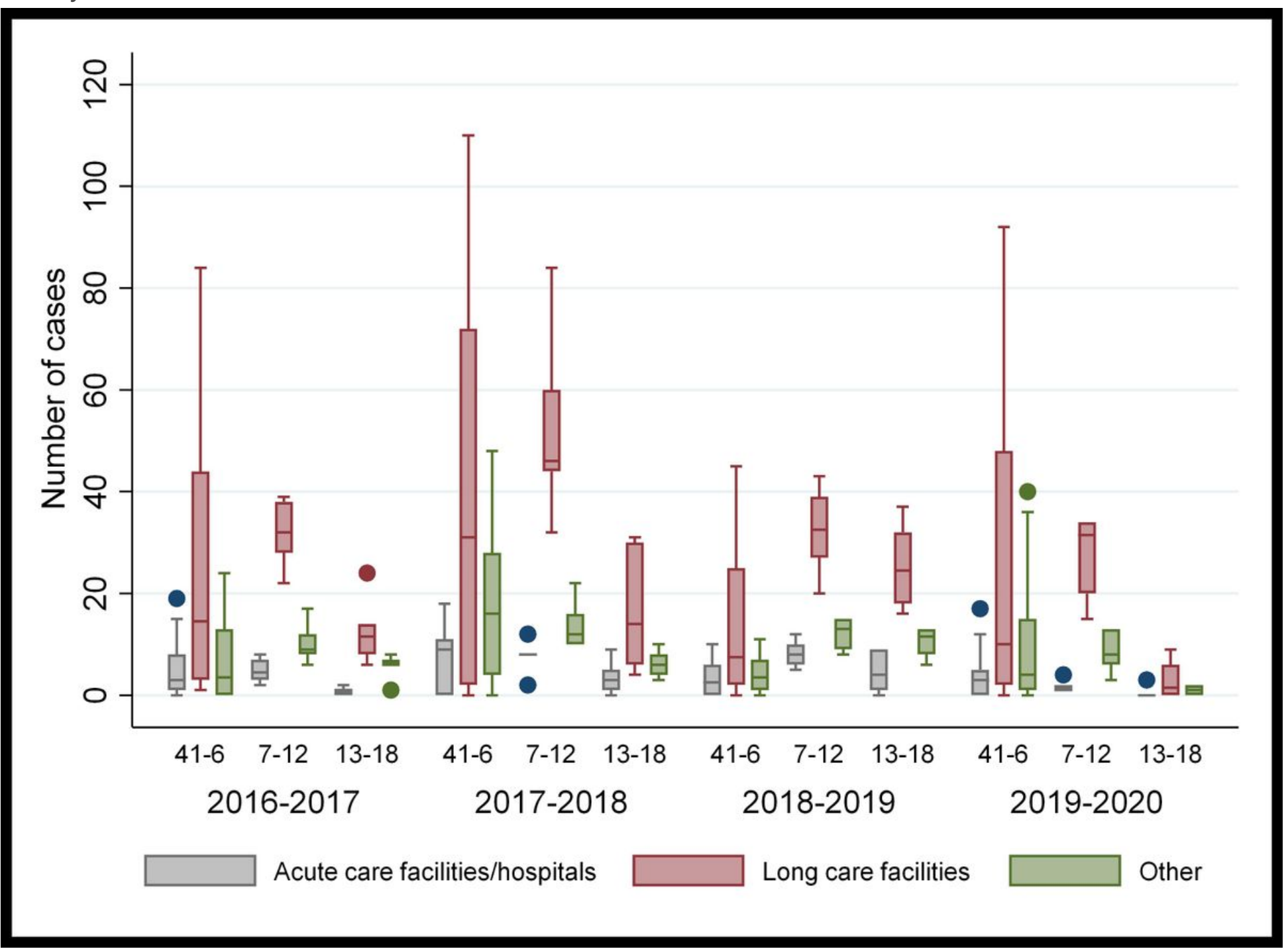

Figure 2

Box-plot of the number of positive influenza B tests in Canada divided by weekly timeframe for four influenza seasons, 2016-17 to 2019-20. 


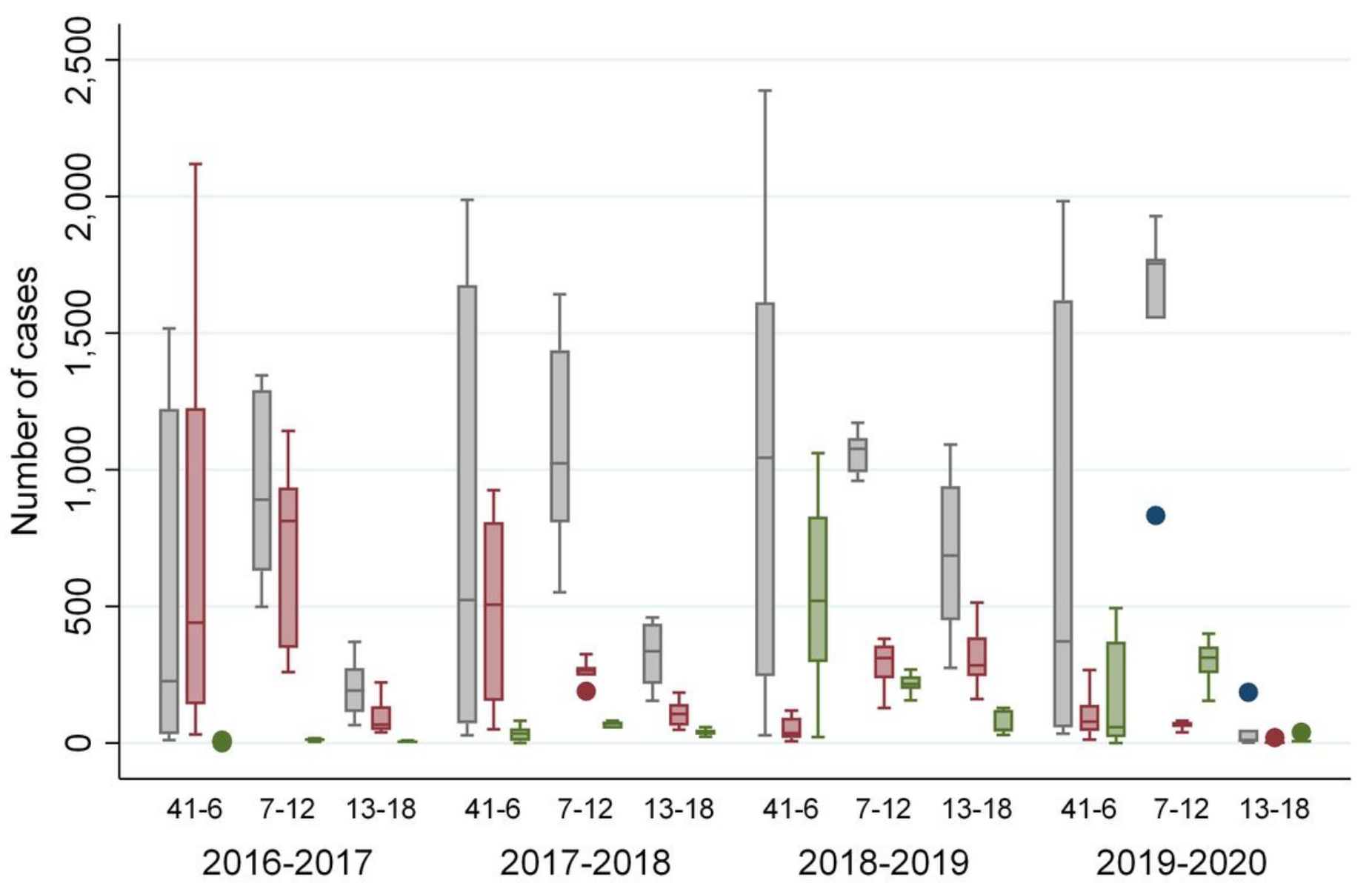

A (Unsubtyped)

$A(H 3 N 2)$

$A(H 1 N 1)$

Figure 3

Box-plot of the number and location of new influenza outbreaks in Canada by weekly timeframe for four influenza seasons, 2016-17 to 2019-20.

Due to technical limitations, Figure 4 is only available as a download in the supplementary files section

Figure 4

\section{Supplementary Files}

This is a list of supplementary files associated with this preprint. Click to download.

- FinallnfluenzaFigure4.xlsx 